\title{
Las asignaturas optativas de formación profesional en la planificación curricular de pregrado
}

\author{
Optative professional training courses towards higher \\ education curricula design
}

María F. Compte Guerrero*

\section{Introducción}

Las universidades ecuatorianas se encuentran en un momento histórico para la educación superior debido a la aprobación de una nueva "Ley Orgánica de Educación Superior"' (LOES), que tiene como principal objetivo transformar y regular a las instituciones de estudios superiores, en todos los aspectos: académico, administrativo, financiero, laboral, etcétera.

Los cambios que actualmente pretende implementar el gobierno ecuatoriano en las universidades implican y demandan la generación de una serie de reglamentos que deben contribuir a reordenarlas, reorganizarlas y regularlas bajo los lineamientos que los organismos pertinentes dicten y a las normativas internas que las propias universidades establezcan para complementar la legislación estatal.

En este contexto de reformas a la educación superior, inédito para las universidades ecuatorianas que están actualmente sometidas a procesos de evaluación institucional y acreditación de carreras, y en el proceso de elaboración del nuevo Reglamento de Régimen Académico por parte del Consejo de Educación Superior, el tema del diseño de un programa de asignaturas optativas como parte de un currículo de educación superior pretende convertirse en una importante contribución a la etapa de diseño mesocurricular de una carrera universitaria.

\footnotetext{
* Esta investigación ha sido realizada en el marco académico del Programa de Doctorado "Metodologías para el diseño, evaluación y mejora de planes, proyectos y programas educativos" de la Facultad de Educación, UNED. Arquitecta por la Universidad Católica de Santiago de Guayaquil, Magister en Gerencia y Liderazgo Educacional por la Universidad Técnica Particular de Loja. Profesora Titular de la Facultad de Arquitectura y Diseño de la Universidad Católica de Santiago de Guayaquil.

1 La LOES fue aprobada por la Asamblea Nacional ecuatoriana el 12 de octubre de 2012.
} 
El problema actual radica en que la reglamentación vigente indica la obligatoriedad de la existencia de estas asignaturas optativas en los planes de estudio de todas las carreras de las instituciones de educación superior ecuatorianas, pero no especifica las pautas para su definición y conformación, por lo que las universidades se han visto abocadas a organizarlas e implementarlas según su criterio particular, lo que muchas veces ha causado que estas asignaturas no respondan totalmente a las necesidades de formación académico-profesional que, principalmente, requiere el sector de la profesión. Es por esto que se hace imperiosa la necesidad de definirlas, diseñarlas, estructurarlas y organizarlas para su implementación y sobre todo para que la determinación de su contenido responda a las demandas reales del quehacer de las profesiones.

\section{El diseño y la planificación curricular}

El proceso de planificación curricular de estudios superiores comprende varias etapas: "análisis previo, diseño curricular, aplicación curricular y evaluación curricular" (Mastromatteo, 2005: 21); en la etapa de diseño curricular "se especifica los fines y objetivos educacionales con base en el análisis previo, se diseñan los medios (contenidos y procedimientos) y se asignan los recursos humanos, materiales informativos, financieros, temporales y organizativos, con la idea de lograr estos fines" (2005: 21).

Considerando este proceso, se puede afirmar que la planificación curricular que corresponde efectuar en toda institución de educación superior para el funcionamiento pleno de sus carreras, debe responder y dar solución a problemas de índole social, político, económico, a más de los educativo-formativos propiamente dichos, para que sus planes de estudio estén actualizados, contextualizados y acordes a las tendencias disciplinares, a las necesidades y demandas reales del mercado laboral donde se desempeñarán sus graduados.

Tomando en cuenta lo que indica Mastromatteo respecto a que las bases curriculares "constituyen el diagnóstico de la situación social que requiere de una intervención educacional para formar los profesionales demandados" (2005: 25), las cuales comprenden la formación que las instituciones de educación superior deben dar a los futuros profesionales, determinada por el conocimiento 
del ejercicio de la profesión, las demandas sociales del mercado laboral de la misma y sus proyecciones.

El diseño curricular implica la estructuración de un plan de estudio que constituye "el conjunto de experiencias de enseñanza-aprendizaje y contenidos cursados en una carrera, para adquirir las competencias profesionales y las características de desempeño, que forme profesionales aptos para responder a las necesidades sociales en general y del campo profesional en particular" (Mastromatteo, 2005: 55).

El diseño curricular de un determinado plan de estudios, por ende, comprende un proceso compuesto de las siguientes fases:

a. El estudio de la realidad social y educativa (aportaciones científicotecnológicas de la comunidad, aspectos socioeconómicos y culturales, planes de estudio vigentes, instituciones e individuos);

b. El establecimiento de un diagnóstico y un pronóstico con respecto a las necesidades sociales;

c. Elaboración de una propuesta curricular como posibilidad de solución de la necesidades advertidas que incluya la selección y determinación de un marco teórico, el diseño de programas y planes de estudio y la elaboración de recursos didácticos, $\mathrm{y}$

d. La evaluación interna y externa de la propuesta. (Aguilar y VargasMendoza, 2011: 55)

Antes de diseñar e implementar un plan de estudio, se deben seguir los siguientes pasos para lograr un correcto y eficiente diseño:

- Definir los campos de estudio y áreas académicas que componen la malla curricular. ${ }^{2}$

- Diseñar los programas educativos que lo componen: de optativas, de prácticas preprofesionales, de formación humanística, con sus respectivos objetivos, contenidos y sistema de evaluación.

- Determinar los objetivos, general y específicos, los resultados de aprendizaje, los contenidos y la forma de evaluación de cada una de las asignaturas.

2 Una malla curricular es una estructura compuesta por los niveles y ciclos de estudios, las asignaturas que conforman un plan de estudios, los créditos académicos por asignatura y totales. 
- Definir la forma organizativa de la actividad docente: tipos de clases (presenciales, semipresenciales, virtuales), métodos y medios de enseñanza, créditos y horas asignadas a cada asignatura.

Los programas que forman parte de un plan de estudio deben estar compuestos por asignaturas integradas en una estructura o malla curricular que ha de responder a los objetivos, metas y perfil profesional declarados en el plan de estudio respectivo. Según la Clasificación Internacional Normalizada de la Educación (CINE) 2011, de la UNESCO, un programa educativo es:

Un conjunto o secuencia de actividades educativas coherentes diseñadas y organizadas para lograr un objetivo predeterminado de aprendizaje o realizar un conjunto especifico de tareas educativas a lo largo de un periodo sostenido. Dentro de un programa educativo, las actividades pueden estar estructuradas en torno a subcomponentes que se conocen por distintos nombres en los países, tales como "cursos", "módulos", "unidades" o "asignaturas". Un programa puede estar organizado en base a componentes no habitualmente considerados cursos, unidades o módulos, por ejemplo, actividades lúdicas, prácticas o pasantías laborales, proyectos de investigación y preparación de disertaciones (2011: 81).

Para Ruiz Echevarría, Asesora de la Dirección de Formación de Profesionales M.E.S., una asignatura es "una agrupación de contenidos de una rama del saber, ordenados de una forma lógica y pedagógica, que posibilitan el cumplimiento de los objetivos generales formulados para la misma, los que se encuentran a su vez vinculados con los objetivos generales de la disciplina a la que pertenece" (1996: 10). Las asignaturas, por lo tanto, son el componente básico de la estructura de un currículo académico, constituyéndose así en la base de los planes de estudio y de las mallas curriculares de las carreras de pregrado o posgrado. Toda asignatura debe contener objetivos generales educativos e instructivos; el contenido específico detallado en unidades de estudio expresado en un sistema de conocimientos, habilidades y resultados de aprendizaje; la forma de evaluación y, el número total de créditos y horas asignados a dicha asignatura.

Las asignaturas de una malla curricular pueden ser obligatorias y optativas, estas últimas son de formación general y de formación profesional; las obliga- 
torias son las que tienen contenido fijo en la malla curricular y las optativas de formación profesional son las correspondientes a los conocimientos que puede elegir el estudiante y que constituyan valor agregado al plan de estudio, con la finalidad de ampliar su formación académico-profesional.

\section{Las asignaturas optativas de formación profesional}

Según lo establecido en las "Normas para la administración de las asignaturas electivas de la Facultad Experimental de Ciencias y Tecnología de la Universidad de Carabobo, Venezuela", la finalidad de las asignaturas de formación profesional es "brindar al estudiantado tópicos de relevancia académica de actualidad y trascendencia, con una profundidad que le permita la cimentación de sus conocimientos básicos adquiridos a través de toda su carrera, y la especialización, profundización y complementación de un aspecto de la ciencia o profesión" (2008: 1). Los programas de este tipo de asignaturas deben ser diseñados con flexibilidad en los tópicos que se imparten.

Para las universidades europeas, pertenecientes al "Espacio Europeo de Educación Superior" (EEES), ${ }^{3}$ la temática de las asignaturas optativas debe estar referida a la propia titulación y son de libre oferta a criterio de cada universidad para que el estudiante escoja un grupo que complete el número de créditos exigidos. Siguiendo estos lineamientos europeos, España expidió el Real Decreto 1339/2007, en el que se regula y marca directrices para la distribución de los "European Credit Transfer System" (ECTS), ${ }^{4}$ estableciéndose que los planes de estudios de Grado "contendrán toda la formación teórica y práctica que el estudiante deba adquirir: aspectos básicos de la rama de conocimiento, materias obligatorias u optativas, seminarios, prácticas externas, trabajos diri-

3 El Espacio Europeo de Educación Superior fue creado a partir de la Declaración de Bolonia en 1999 con el objetivo principal de unificar criterios en la planificación curricular de los países que la suscribieron para facilitar la movilidad estudiantil y el reconocimiento de títulos en el espacio europeo de educación superior.

4 European Credit Transfer System, es el Sistema Europeo de Transferencia de Créditos que han adoptado las universidades europeas adscritas al EEES. El cálculo de un ETCS es igual a veinticinco horas de trabajo de un estudiante. 
gidos, trabajo de fin de Grado u otras actividades formativas" e incluso en los estudios de Máster están incluidas las asignaturas optativas. En cumplimiento a este Real Decreto, las universidades españolas distribuyeron los créditos de los estudios de Grado otorgándoles entre doce a treinta créditos a las asignaturas optativas de la titulación, es decir, de las que su contenido está directamente relacionado con la carrera. Pero la reglamentación general para las universidades españolas no detalla cómo deben organizarse este tipo de asignaturas, cuáles son sus objetivos y una posible orientación de cómo estructurarlas, por lo que cada universidad las regula internamente.

Igualmente sucede en las universidades latinoamericanas, sean chilenas, argentinas, colombianas, etcétera, que contemplan asignaturas de libre elección u optativas en sus planes de estudio, asignándoles entre quince a treinta créditos, y cada institución de educación superior las estructura y organiza de acuerdo a su reglamentación interna.

En las instituciones de educación superior ecuatorianas las asignaturas optativas de formación profesional deben formar parte del pensum académico de pregrado de manera obligatoria desde el año 2009 cuando el entonces "Consejo Nacional de Educación Superior" (CONESUP) 5 expidió el Reglamento de Régimen Académico aún vigente, con el objetivo que los estudiantes actualicen y profundicen sus conocimientos. Acogiendo esa disposición, cada universidad reformó los planes de estudio de sus carreras de acuerdo a este nuevo requerimiento de incluirlas en sus mallas curriculares, muchas veces adaptando lo que ya tenían institucionalizado, como seminarios, talleres, menciones, etcétera.

La normativa ecuatoriana vigente solo indica que las asignaturas optativas de formación profesional deben estar presentes en una malla curricular, pero no especifica cómo diseñarlas e implementarlas; su existencia implica diseñar un programa que contenga objeto; objetivo; contenido; la metodología a seguir para su determinación; método, forma y medios de enseñanza; además de un sistema que permita evaluarlo periódicamente.

5 El CONESUP (Consejo Nacional de Educación Superior) fue reemplazado por el Consejo de Educación Superior (CES) a partir de la vigencia de la LOES (Ley Orgánica de Educación Superior aprobada por la Asamblea Nacional el 12 de octubre de 2010. 
Haciendo una investigación y análisis sobre las asignaturas optativas de formación profesional en las universidades ecuatorianas, se puede observar que la mayoría las incluyen en el pensum académico de sus carreras, pero no cuentan con un programa específico para ellas, lo que ocasiona que no se sepa a ciencia cierta si estas asignaturas están contribuyendo a la formación académica de los estudiantes y sobre todo, a su futuro desenvolvimiento en el mercado laboral de su profesión.

\section{La investigación}

La investigación ${ }^{6}$ realizada, base de este estudio, tuvo como objetivo principal la construcción de un modelo metodológico para el diseño, implementación y evaluación de un programa de asignaturas optativas de formación profesional aplicable a las carreras de arquitectura de las universidades de la ciudad de Guayaquil, pertenecientes al sistema de educación superior ecuatoriano, para lo cual se utilizó el Modelo de Evaluación del Programa en su momento inicial de Pérez Juste, ${ }^{7}$ que toma en cuenta tres dimensiones: la calidad intrínseca del programa, la adecuación al contexto, y la adecuación a la situación de partida.

La calidad intrínseca del programa se refiere a su contenido, al "documento técnico en que toma cuerpo el programa” (Pérez Juste, 2006: 219), es decir, todos los elementos que forman parte de él, como: objetivos, contenido, medios, recursos, etcétera, que lo configuran y regulan; las más importantes son las metas, que deben responder a las necesidades que pretenden ser cubiertas y satisfechas con el programa, y los medios y recursos que deben ser "adecuados y suficientes" (2006: 221). La calidad técnica del programa se refiere a si este

6 Trabajo de investigación efectuado por la Mgs. María Fernanda Compte Guerrero para la Tesis doctoral "Modelo metodológico para el diseño, implementación y evaluación de un programa de asignaturas optativas aplicable a las carreras de arquitectura de las Universidades de la ciudad de Guayaquil, pertenecientes al sistema de educación superior ecuatoriano".

7 Ramón Pérez Juste, catedrático de la Facultad de Educación de la UNED, ha desarrollado un modelo de evaluación de programas educativos basado en cuatro momentos: Inicial: el programa en cuanto tal, Procesual: el proceso de implantación del programa, Final: el programa en sus resultados, Institucionalización de la evaluación del programa. 
se ajusta a requerimientos y exigencias técnicas, y la evaluabilidad mide si el programa puede ser objeto de evaluación.

La adecuación al contexto es otro de los criterios del modelo en su momento inicial, que considera "las necesidades, carencias y expectativas de los destinatarios, y a las características del lugar y el momento en el que el programa será aplicado" (2006: 222-223). La adecuación a las circunstancias o la viabilidad del programa debe ser evaluada a base de sus objetivos, que deben ser viables y cumplibles ya que su incumplimiento conlleva al fracaso del programa o a que este no responda a las necesidades detectadas inicialmente.

Los métodos y técnicas utilizadas fueron de índole cualitativo ya que la investigación estuvo enmarcada en el paradigma interpretativo que "recoge lo idiográfico, lo experiencial, lo trascendental, lo holístico y lo personal" (Martínez Mediano, 1996: 18); la recopilación de información se la realizó mediante revisión bibliográfica y documental, y las entrevistas en profundidad de tipo semiestructurada y grupos focales se efectuaron a: los principales directivos de las carreras de arquitectura de las universidades de Guayaquil, a expertos en diseño curricular, a representantes de instituciones y empresas públicas y privadas del mercado laboral de la profesión, a graduados y estudiantes de los últimos semestres de la carrera, con la finalidad de indagar sobre los criterios y consideraciones académicas que deben estar presentes en el diseño de un programa de asignaturas optativas de formación profesional, sobre la estructuración de este tipo de asignaturas en universidades extranjeras, sobre cómo estructuran y plantean las asignaturas optativas de formación profesional las cuatro carreras de arquitectura de Guayaquil, la visión del mercado laboral sobre el tipo de profesionales que se requieren.

\section{Los resultados y la propuesta}

Luego del análisis de contenido y documental efectuado a la información recopilada, se establecieron los criterios que deben estar presentes al momento de diseñar e implementar un programa de asignaturas optativas de formación profesional para una carrera de pregrado, ya que aunque la investigación se centró en las carreras de arquitectura de la ciudad de Guayaquil, se llegó a la 
conclusión de que el modelo construido para el diseño del programa puede ser utilizado en cualquier tipo de carrera perteneciente al sistema de educación superior ecuatoriano, ya que es un modelo genérico debido a que está conformado por variables y elementos que siempre deben estar presentes en la programación académica de una institución de educación superior.

Ya que hasta la fecha los organismos estatales pertinentes no han dictado lineamientos y regulaciones claras y específicas para la conformación de un programa de asignaturas optativas de formación profesional que debe formar parte de los planes de estudio de todas las carreras universitarias, se proponen los siguientes conceptos, criterios y parámetros que pueden servir de guía para la estructuración de dicho programa.

Antes de diseñar el programa, es necesario realizar un diagnóstico del estado del arte del campo laboral de la profesión para determinar sus necesidades y demandas, y así tener la certeza de que el programa va a contribuir a cubrir dichas necesidades con la formación académica de los futuros profesionales. Este diagnóstico se lo puede realizar investigando la actividad laboral de los graduados de la carrera y a los directivos de las empresas e instituciones, pública y privadas, donde ellos laboran.

Los resultados del diagnóstico van a dar como resultante el perfil profesional que requiere el sector de la profesión, ${ }^{8}$ el mismo que debe ser contrastado con el perfil profesional ${ }^{9}$ que consta en el plan de estudio de la carrera para determinar qué áreas o campos están siendo demandados y que no están dentro del mismo; este nuevo perfil profesional, llamado Producto, ${ }^{10}$ es el que realmente responderá a las necesidades formativas y disciplinares del mercado laboral. Una vez determinado el Perfil Profesional Producto, es posible establecer los conocimientos que pueden ser abarcados a través de las asignaturas optativas de formación profesional y luego diseñar el programa respectivo.

8 El Perfil Profesional que constituye el conjunto de características que son propias de los profesionales de una carrera, se denomina Real. "Es el futuro probable” (García, 58).

9 El Perfil Profesional que forma parte del plan de estudio de la carrera se denomina Ideal, y "se refiere al cómo se cree que debería ser el profesional. Configura un objeto anhelado" (García, 58).

10 El Perfil Profesional Producto "es la propuesta considerada factible de ser implantada y que rediseña el perfil académico, otorgando nuevas competencias al graduado y planeando una nueva estructura curricular. Es el futuro posible" (García, 59). 
A continuación se proponen criterios y características para la conformación del programa:

- El objetivo principal es complementar la formación académico-profesional de los estudiantes en los aspectos que demande el mercado laboral y las tendencias disciplinares y dinámicas del sector de la profesión, reforzándola en temáticas afines a la carrera que no están incluidas de manera permanente en la malla curricular, permitiendo a los estudiantes la profundización de aspectos concretos del conocimiento, y otorgando flexibilización al currículo.

- Las asignaturas que lo conforman, pueden servir de relación entre la formación universitaria y la práctica profesional-laboral, y a la orientación de los estudiantes para sus futuros estudios de cuarto nivel.

- Debe ser prospectivo y estar conectado con las líneas de investigación de la carrera y con la vinculación con la comunidad, actividades sustantivas de una institución de educación superior, considerando que las disciplinas que los sustentan están en un constante cambio, así como también las demandas del sector de la profesión.

- La oferta de las asignaturas optativas de formación profesional debe ser variada y constante para que los estudiantes tengan un abanico donde elegir los contenidos según sus intereses profesionales.

- El contenido de las asignaturas del programa debe responder principalmente a dos vertientes: las tendencias de la profesión a nivel nacional y mundial, y lo que los estudiantes demandan de conocimientos.

- El programa debe estar relacionado con las áreas académicas de la carrera y sus líneas de investigación.

- Estas asignaturas deben estar ubicadas a partir del nivel básico-específico de la malla curricular, es decir, a partir del cuarto semestre de la carrera que es cuando los estudiantes ya han adquirido ciertos conocimientos y competencias previas a la mención que escogerán.

- Las autoridades académicas que diseñarán el programa deberán asesorarse por un grupo de profesionales y representantes de los gremios de la profesión que analice lo que el sector demanda. 
- $\quad$ El porcentaje de créditos totales de las asignaturas pertenecientes al programa debe estar en un rango del $5 \%$ al $10 \%$, es decir, de doce a veinticuatro créditos, en una malla curricular de 245 créditos. ${ }^{11}$

- Estas asignaturas deben tener un valor académico de tres o cuatro créditos, lo que en horas es de 48 a 64 horas. ${ }^{12}$

- El programa debe contar con un modelo de evaluación para poder determinar, periódicamente, si está cumpliendo o no con los objetivos que motivaron su creación.

- Las autoridades académicas de la carrera respectiva deben brindar consejería académica a los estudiantes para orientarlos en la selección de los contenidos de las asignaturas optativas ofertadas por la carrera.

- Dada la característica flexible de las optativas de formación profesional, su contenido puede ser cambiado en cualquier momento en que lo amerite de acuerdo a las demandas y tendencias del sector laboral.

- El programa debe ser revisado y actualizado al término de duración de una cohorte. ${ }^{13}$

- El programa debe estar vinculado a los campos de estudio de la malla curricular, ser de libre elección por parte de los estudiantes y estar relacionado con el perfil profesional producto de la carrera.

- Puede formar una concentración de contenidos tendientes a lograr una especificidad en el pregrado a través de las menciones o certificaciones de competencias en un determinado campo de estudio de la profesión.

\section{Conclusiones}

Es necesario que universidades ecuatorianas cuenten con un programa estructurado con objetivos, lineamientos, contenidos, metas, recursos y medios,

11 El Reglamento de Régimen Académico vigente a la fecha de este artículo establece que una carrera de pregrado debe tener 245 créditos en total.

12 El citado reglamento también indica que un crédito es igual a dieciséis horas de efectivas de clases y dieciséis horas de aprendizaje autónomo.

13 Una cohorte es un periodo académico, generalmente del tiempo de duración de la carrera, cuatro o cinco años según sea el caso. 
para organizar y estructurar las asignaturas optativas de formación profesional, que por disposiciones reglamentarias de los organismos estatales que las regulan, forman parte del plan de estudios y por ende de la malla curricular de todas las carreras.

En las reglamentaciones vigentes, estatales e institucionales, simplemente se indica la obligatoriedad de la existencia de este tipo de asignaturas pero no se dictan los lineamientos necesarios para su conformación, lo que ha llevado a las universidades a incluirlas dentro de su currículo, sin un estudio previo de las variables que deben ser analizadas para la conformación de un programa que responda a las necesidades y demandas académicas, sociales y políticas de la profesión.

La inclusión de la visión del mercado laboral de la profesión es muy importante ya que los estudiantes, al graduarse, van a ejercer la profesión en ese mercado influenciado por las demandas sociales y las tendencias disciplinares actuales; es por esto que la reglamentación pertinente al diseño curricular de las carreras debe proponer una metodología a seguir para el diseño del programa, que incluya las variables operacionales que deben estar siempre presentes en una institución de educación superior y sus programas educativos, y toda carrera cuenta con un mercado laboral o sector de la profesión donde sus graduados ejercerán poniendo en práctica los conocimientos adquiridos en la universidad.

\section{Bibliografía}

AGUILAR, Jorge. et al.

2011 Planeación educativa y diseño curricular: un ejercicio de sistematización. Notas: Boletín Electrónico de Investigación de la Asociación Oaxaqueña de Psicología A.C. Volumen 7. Número 1. México. Disponible en: http://www. conductitlan.net/notas_boletin_investigacion/140_planeacion_educativa_ curriculum.pdf. [Fecha de consulta: abril 30 de 2012].

BOLONIA: ESPACIO EUROPEO DE EDUCACIÓN SUPERIOR.

Ministerio de Educación, Gobierno de España.

http://www.educacion.es/boloniaeees/estructura.html. 
COMPTE, María Fernanda

2013 Evaluación académica del aporte de las asignaturas electivas al perfil profesional de los arquitectos de la UCSG del periodo 2004-2010 y su relación con la demanda del sector de la profesión. Guayaquil. Dirección de Publicaciones de la UCSG.

COMPTE, María Fernanda

2011 Diseño de modelo metodológico de intervención para la conformación del programa de asignaturas optativas para el currículo de arquitectura de la $U C S G$. Guayaquil. Informe final del trabajo de investigación realizado para el Sistema de Investigación y Desarrollo SINDE de la UCSG.

FACULTAD EXPERIMENTAL DE CIENCIAS Y TECNOLOGÍA DE LA UNIVERSIDAD DE CARABOBO.

2008 Normas para la administración de las asignaturas electivas. Venezuela. Disponible en: http://portal.facyt.uc.edu.ve/downloads/reglamentos/norm electivas.pdf. [Fecha de consulta: febrero 16 de 2010].

GARCÍA, Guillermo

1996 El diseño de perfiles profesionales prospectivos y análisis curricular en educación superior. Guayaquil. Universidad Católica de Santiago de Guayaquil.

LEY ORGÁNICA DE EDUCACIÓN SUPERIOR. Quito, R.O. N 298, 12-X-2010.

MARTÍNEZ MEDIANO, Catalina

1996 Evaluación de programas educativos. Investigación Evaluativa. Modelo de Evaluación de Programas. Madrid. UNED.

MASTROMATTEO, Estela

2005 Bases, fundamentos y perfil profesional. Aporte para el cambio curricular de la EBA-UCV. Tesis de maestría. Caracas. Universidad Central de Venezuela. Disponible en: eprints.rclis.org/bitstream/10760/7672/1/tesismsc.. pdf. [Fecha de consulta: abril 30 de 2012].

PÉREZ JUSTE, Ramón. et al.

2012 Métodos y Diseños de investigación en Educación. Madrid. UNED.

PÉREZ JUSTE, Ramón

2006 Evaluación de programas educativos. Madrid. La Muralla.

REAL DECRETO 1339

2007 Boletín Oficial del Estado. BOE. núm. 260. España.

REGLAMENTO DE RÉGIMEN ACADÉMICO.

2009 Quito. CONESUP. 
RUIZ ECHEVARRÍA, Hilda

1996 "El programa de la asignatura: un modelo del proceso docente educativo". Revista Pedagogía Universitaria del Ministerio de Educación de Cuba. 1.

\section{UNESCO}

2011 Clasificación Internacional Normalizada de la Educación 2011. Obtenido el 6 de abril de 2012 desde http://unesdoc.unesco.org/ images/0021/002116/211619s.pdf. 